\title{
BASIC RESEARCH FOR NEW LASER GLASSES
}

T. IZUMITANI

HOYA GLASS WORKS, LTD.

A few years ago, we developed LSG-91H laser glass which has about the same gain coefficient as ED-2. In order to obtain glasses with higher gain coefficients, we investigated on lasing properties of glass, ie, we measured the absorption spectrum and the fluorescent decay time, then estimated the gain coefficient of a Nd-doped laser glass.

CALCULATIONS

The fluorescent intensity is given by eqn. (1).

$$
I_{F}^{1.06}=A_{1.06} \Delta \mathrm{N}
$$

, where $A_{1.06}$ is Einstein's spontaneous emission coeff. at $1.06 \mu$ and $\Delta \mathrm{N}$ is inversion population. Inversion population is given by eqn. (2) for the steady state of 3-level system.

$$
\Delta \mathrm{N} \doteqdot \mathrm{N}_{0} \mathrm{~B} \tau
$$

, where $\mathrm{N}_{0}$ is the number of $\mathrm{Nd}$ ions at ground level; $\mathrm{B}$, induced absorption coefficient from the ground state to the higher levels; $\mathrm{ta}$, apparent life time from the 3rd lasing energy level to the 2nd level.

$A_{1.06}$ can be calculated using absorption spectrum at $0.88 \mu\left({ }^{4} \mathrm{I}_{9} / 2 \rightarrow{ }^{4} \mathrm{~F}_{3} / 2\right)$ and the ratio of the fluorescent intensity at $1.06 \mu$ and $0.88 \mu\left({ }^{4} \mathrm{~F}_{3} / 2 \longrightarrow{ }^{4} \mathrm{I}_{11 / 2}\right.$ and ${ }^{4} \mathrm{~F}_{3 / 2} \longrightarrow{ }^{4} \mathrm{I}_{9} / 2$ ).

$$
\left.\begin{array}{l}
A_{1.06}=r \cdot A_{0.88}=\frac{I_{1.06}}{I_{0.88}} \cdot A_{0.88} \\
A_{0.88}=\frac{8 \pi^{2}}{\lambda} \cdot \frac{g_{i}}{g_{j}} \cdot \frac{1}{N_{0}} \int \mathrm{KdV}
\end{array}\right\}
$$

$A_{1.06}$ coefficient is related to the quantum efficiency $\eta_{3}$ and apparent life time $\tau a$

$$
A_{1.06}=\eta_{3} / \tau_{a}
$$


Inversion population $(\Delta \mathrm{N})$ at 3 rd level is given by eqn. (2) and experimentally obtained from the fluorescent intensity and A-coefficient at $0.88 \mu$ using eqn. (5).

$$
\Delta \mathrm{N}=I_{\mathrm{F}}^{0.88} / \mathrm{A}_{0.88}
$$

Cross section for stimulated emission $(\sigma)$ is given by the Landenburg-Füchtbauer equation and obtained experimentally from A-coefficient and the fluorescent. half-width $(\Delta \lambda)$.

$$
\sigma_{1.06}=\frac{\lambda^{4} A_{1.06}}{4 \pi^{2} \Delta \lambda_{1.06}} \propto_{A_{1.06}} / \Delta \lambda_{1.06}
$$

Gain coefficient (G) is given as follows:

$$
\mathrm{G}=\sigma \Delta \mathrm{N}
$$

From eqn. (5), (6) and (7),

$$
G \propto \frac{A_{1} .06}{\Delta \lambda_{1.06}} \cdot \frac{I_{F}^{1.06}}{A_{1.06}}=\frac{I_{F}^{1.06}}{\Delta \lambda{ }_{1.06}}=I_{P}
$$

Therefore, gain coefficient is estimated from the peak fluorescent intensity at $1.06 \mu$.

\section{EXPERIMENTAL}

Table I shows the glass compositions used. In the lst series the ratio of the content of network former to that of modifier was changed. In the 2nd series, the kind of alkali ions was changed. In the $3 \mathrm{rd}$ and $4 \mathrm{th}$ series, the kind of alkali earth ions was changed. In the 5 th series, the kind of network former was changed. In the 6th series, the kind of alkali earth ions was changed in phosphate glasses.

Fig.l shows the schematic diagram for lifetime measurement. Xe-flash lamp was used as a light source. Fig.2 shows the schematic diagram for fluorescent spectrum measurement. A continuous Xe-lamp was used for the excitement of $\mathrm{Nd}$ ions.

As shown in Fig.3, A-coefficient increases with increasing quantum efficiency. This result is expected from eqn. (4), if lifetime is constant. Although A-coefficient is very high in borate glass, $\Delta \mathrm{N}$ is very small. This means the non-radiative transition probability is too high in borate glasses. As a result, stored 
energy is very small and, therefore, high gain coefficient cannot be expected in borate glasses.

Fig. 4 shows that A-coefficient increases with decreasing lifetime. If the quantum efficiency $\gamma_{3}$ is constant, these results can be expected from eqn. (4).

Fig. 5 shows that inversion population (stored energy) $\Delta \mathrm{N}$ increases apparent lifetime. This result is understandable from eqn. (2).

It is noted in Fig. 6 that the cross section for stimulated emission $(\sigma=A / \Delta \lambda)$ is in inverse relationship to the stored energy. The fact that lasing crystals such as YAG has higher o but lower stored energy than glass, is understandable from this relationship.

Fig. 7 shows the relationship between gain coeff. and apparent lifetime. The gain coefficient increases first and then decreases with decreasing apparent lifetime. This relationship corresponds to the fact that the gain coefficient increases with A-coefficient and decreases with decreasing stored energy, considering the relationships between apparent lifetime and A-coeff. (eqn. (4)) and between lifetime and stored energy (eqn. (2)). Thus, the maximum is expected in the relationship between gain coefficient and apparent lifetime. Fig. 8 shows the relationship between fluorescent intensity and half-width. This figure shows that phosphate glasses have the high fluorescent intensity and narrow half-width, suggesting that they have high gain coefficients.

\section{SUMMARY}

(1) Einstein's A-coefficent at $1.06 \mu$ becomes higher, as quantum efficiency increases and apporent lifetime decreases.

(2) Inversion population (or stored energy) becomes larger as apparent lifetime increases.

(3) It is interesting to note that cross section for stimulated emission(or A-coefficient) and stored energy (or inversion population) are in inversely proportional relationship.

(4) From the view point of A-coefficient and inversion population, some phosphate glasses are considered to be the best host for Nd laser glasses. 


$$
\begin{aligned}
& \text { Table } 1
\end{aligned}
$$

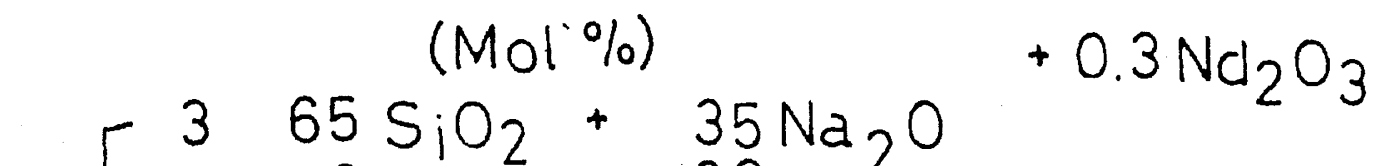

$$
\begin{aligned}
& I\left[\begin{array}{lll}
3 & 65 \mathrm{SiO}_{2}+ & 35 \mathrm{Na}_{2} \mathrm{O} \\
4 & 70 & 30 \\
5 & 75 & 25 \\
6 & 80 & 20
\end{array}\right. \\
& \text { II }\left[\begin{array}{rrr}
8 & 65 \mathrm{SiO}_{2} & +15 \mathrm{Li}_{2} \mathrm{O} \\
9 & & \mathrm{Na}_{2} \mathrm{O} \\
10 & \mathrm{~K}_{2} \mathrm{O}
\end{array}\right. \\
& \text { III }\left[\begin{array}{llr}
11 & 65 \mathrm{SiO}_{2}+30 \mathrm{Li}_{2} \mathrm{O}+ & 5 \mathrm{MgO} \\
13 & & \mathrm{CaO} \\
14 & \mathrm{SrO} \\
14 & \mathrm{BaO}
\end{array}\right. \\
& \text { IV }\left[\begin{array}{rrr}
17 & 65 \mathrm{SiO}_{2}+15 \mathrm{~K}_{2} \mathrm{O}+ & 20 \mathrm{MgO} \\
18 & & \mathrm{CaO} \\
20 & & \mathrm{SrO} \\
\mathrm{BaO}
\end{array}\right. \\
& \nabla\left[\begin{array}{rr}
21 & 67 \mathrm{P}_{2} \mathrm{O}_{5}+15 \mathrm{~K}_{2} \mathrm{O}+1.8 \mathrm{BaO} \\
22 & \mathrm{~B}_{2} \mathrm{O}_{3} \\
23 & \mathrm{SiO}_{2} \\
24 & \mathrm{GeO}_{2}
\end{array}\right. \\
& \text { III }\left[\begin{array}{rr}
25 & 55 \mathrm{P}_{2} \mathrm{O}_{5}+40 \mathrm{MgO}_{2}+5 \mathrm{La}_{2} \mathrm{O}_{3} \\
26 & \mathrm{CaO} \\
28 & \mathrm{SrO} \\
28 & \mathrm{BaO}
\end{array}\right.
\end{aligned}
$$




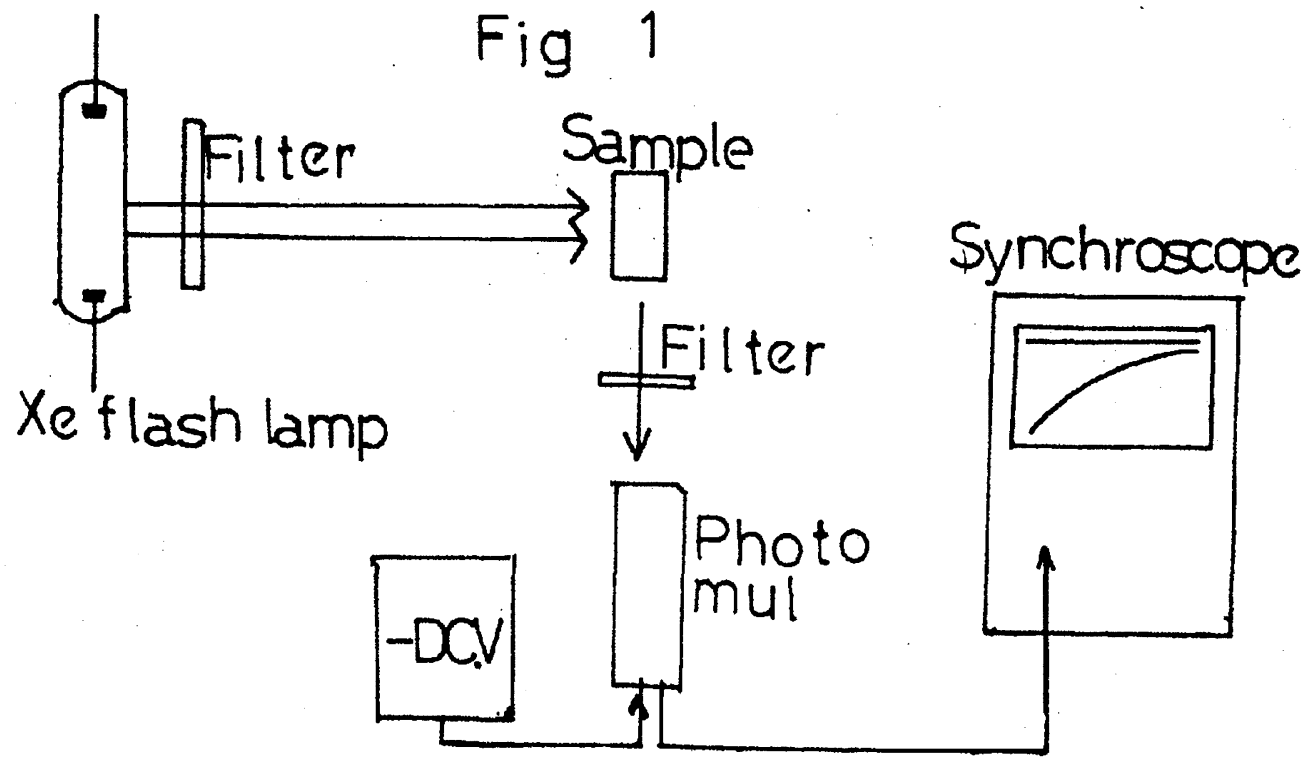

Schematic Diagram for Lifetime Measurement

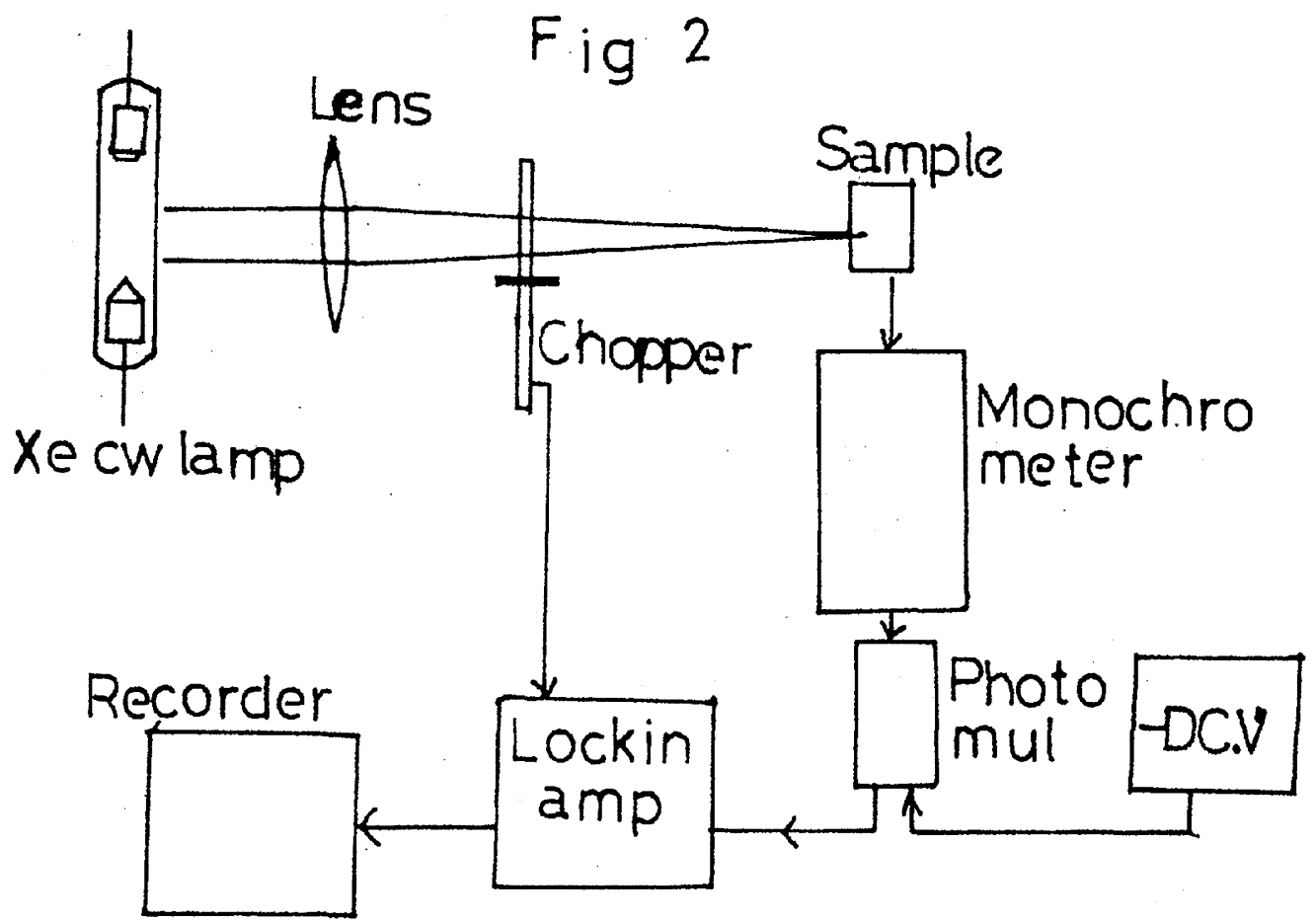

Schematic Diagram for Fluorescent Spectrum Measurement 


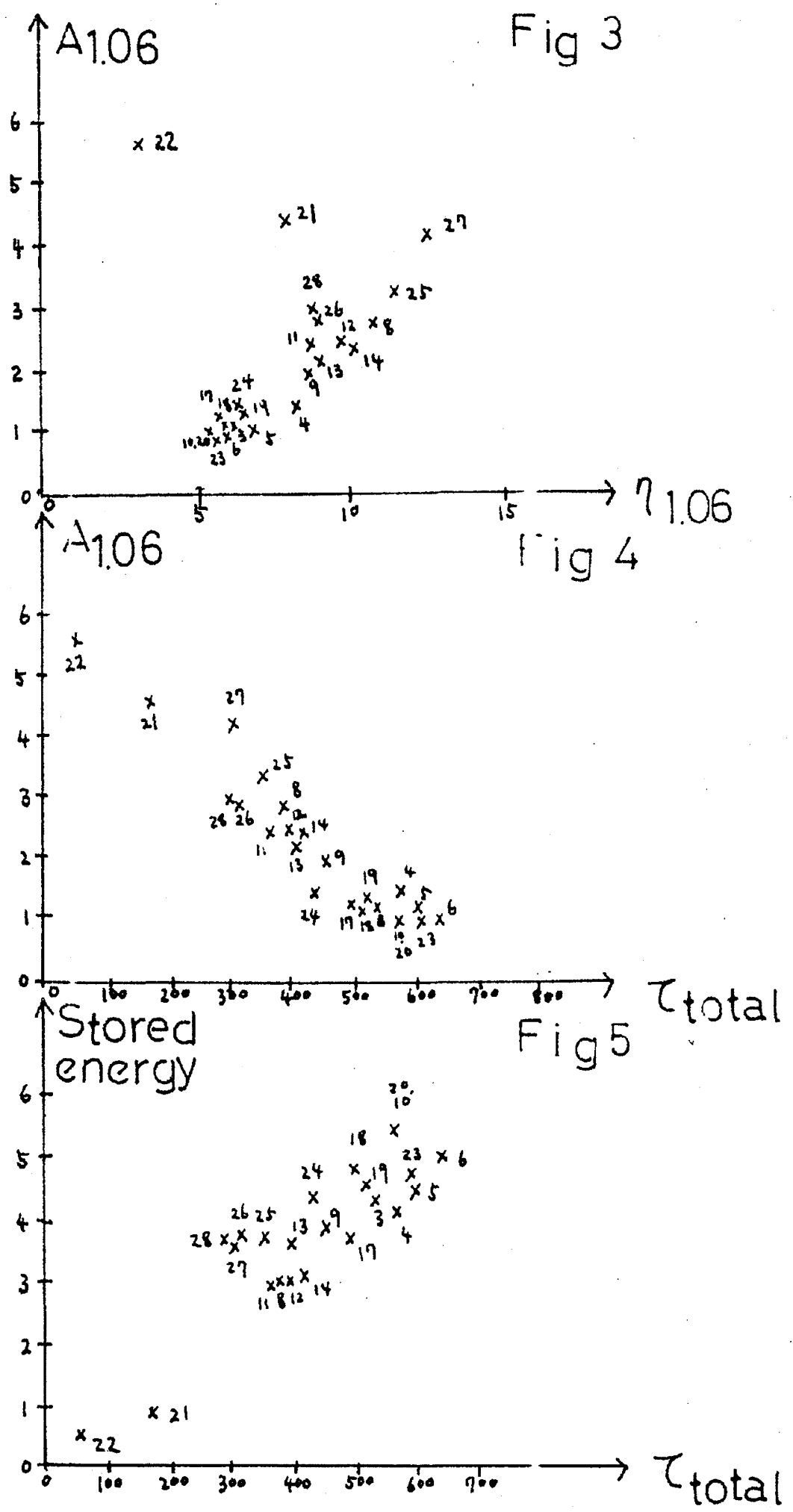




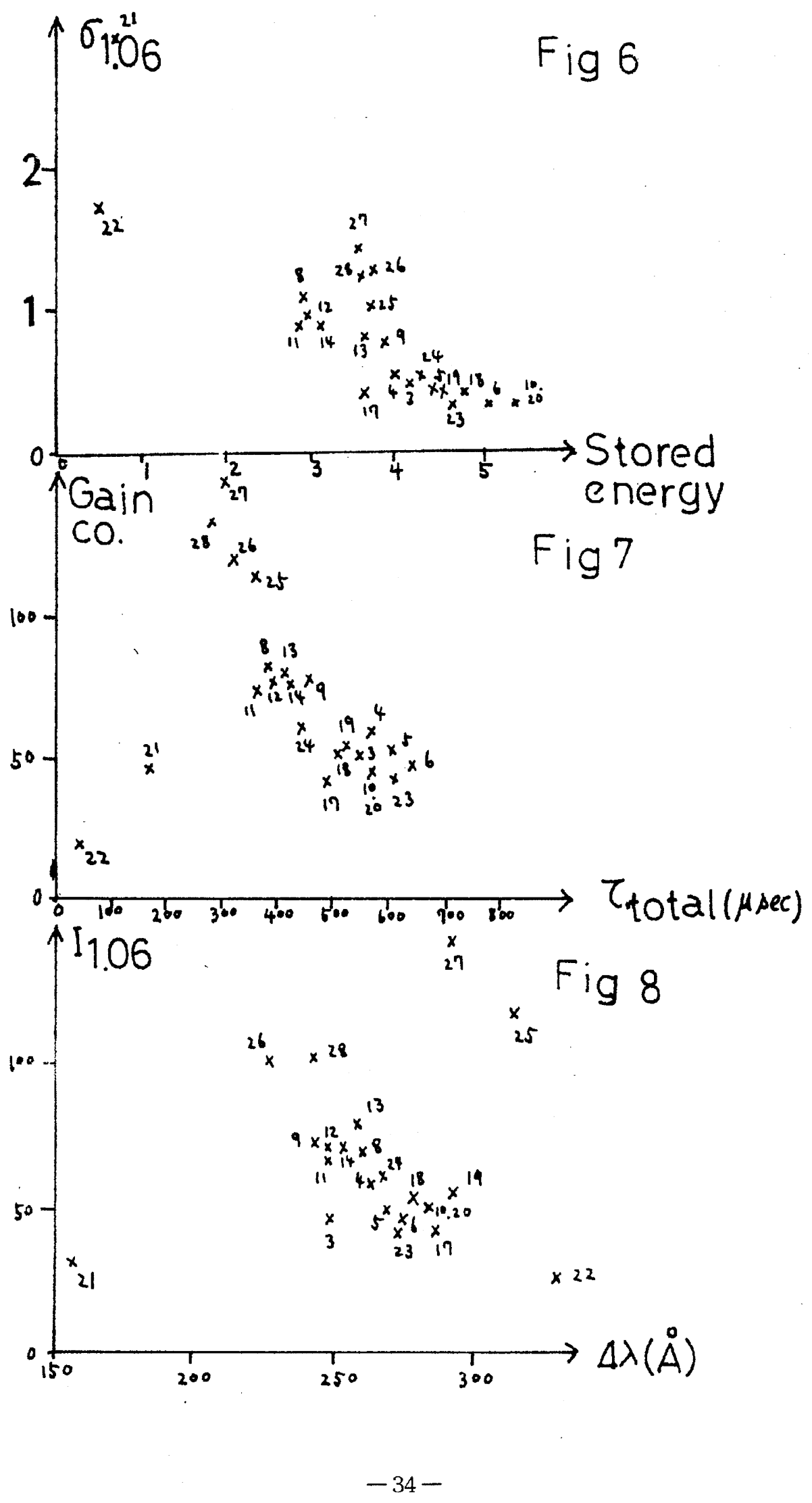

